\title{
Integrated safety and efficacy analysis of once-daily fluticasone furoate for the treatment of asthma
}

Paul M. O'Byrne ${ }^{1,7^{*}}$, Loretta Jacques ${ }^{2}$, Caroline Goldfrad ${ }^{3}$, Namhee Kwon ${ }^{4}$, Michael Perrio ${ }^{5}$, Louisa J. Yates ${ }^{4}$ and William W. Busse ${ }^{6}$

\begin{abstract}
Background: Fluticasone furoate is a once-daily inhaled corticosteroid. This report provides an overview of safety and efficacy data that support the use of once-daily fluticasone furoate $100 \mu \mathrm{g}$ or $200 \mu \mathrm{g}$ in adult and adolescent asthma patients.

Methods: Fourteen clinical studies (six Phase II and eight Phase III) were conducted as part of the fluticasone furoate global clinical development programme in asthma. Safety data from 10 parallel-group, randomised, double-blind Phase II and III studies (including 3345 patients who received at least one dose of fluticasone furoate) were integrated to provide information on adverse events, withdrawals, laboratory assessments, vital signs and hypothalamic-pituitaryadrenal axis function. The efficacy of once-daily fluticasone furoate was evaluated in all included studies.

Results: Once-daily fluticasone furoate $100 \mu \mathrm{g}$ and $200 \mu \mathrm{g}$ safety profiles were consistent with those reported for other inhaled corticosteroids, and both doses consistently demonstrated efficacy versus placebo. In the integrated analysis, no dose-response relationship was observed for the overall incidence of adverse events and there were no significant effects of fluticasone furoate on hypothalamic-pituitary-adrenal axis function.

Conclusion: Once-daily fluticasone furoate $100 \mu \mathrm{g}$ and $200 \mu \mathrm{g}$ had acceptable safety profiles and was efficacious in adult and adolescent patients with asthma. There was no evidence of cortisol suppression at studied doses.

Trial registrations: GSK (NCT01499446/FFA20001, NCT00398645/FFA106783, NCT00766090/112202, NCT00603746/ FFA109684, NCT00603278/FFA109685, NCT00603382/FFA109687, NCT01436071/115283, NCT01436110/115285, NCT01159912/112059, NCT01431950/114496, NCT01 165138/HZA106827, NCT01086384/106837, NCT01134042/ HZA106829 and NCT01244984/1139879).
\end{abstract}

Keywords: Adverse events, Cortisol suppression, Fluticasone furoate, Forced expiratory volume in one second, Inhaled corticosteroid, Integrated analysis, Safety

\section{Background}

Inhaled corticosteroids (ICS) are the mainstay of treatment for all severities of persistent asthma, leading to improved symptom control, improved lung function and quality of life, and reduced asthma mortality [1]. Nevertheless, poorly controlled asthma remains prevalent [2],

\footnotetext{
* Correspondence: obyrnep@mcmaster.ca

${ }^{1}$ Michael G. DeGroote School of Medicine, McMaster University, Hamilton,

ON, Canada

${ }^{7}$ Department of Medicine, Michael G. DeGroote School of Medicine, McMaster University, 1280 Main Street West, Room 3W10, Hamilton ON L8S 4K1, Canada

Full list of author information is available at the end of the article
}

and poor treatment adherence is well documented [1]. Most ICS are administered twice daily; however, improved compliance with once-daily regimens has been demonstrated in mild-to-moderate asthma [3]. A case-control study using the UK General Practice Research Database reported better adherence and reduced costs for patients with asthma who switched to once-daily ICS from twicedaily ICS [4].

The tolerability profile of ICS for asthma treatment is well established. Localised effects, e.g., dysphonia and oral candidiasis, have been reported [1], and prolonged high-dose ICS usage has been associated with adrenal 
suppression, cataracts and glaucoma [1]. Therefore, for a new ICS it is important to assess the safety profile, including potential systemic effects, e.g., cortisol supression.

The ICS fluticasone furoate (FF), administered using the ELLIPTA ${ }^{\circ}$ dry powder inhaler, is a once-daily maintenance treatment for adult and adolescent ( $\geq 12$ years) patients with asthma. FF has higher cellular accumulation and slower efflux compared with other corticosteroids [5], resulting in enhanced lung residency and 24-h efficacy in patients with asthma [6-8]. FF $100 \mu \mathrm{g}$ or $200 \mu \mathrm{g}$ is approved as monotherapy in a number of countries, including the USA [9], and in combination with vilanterol, a novel long-acting beta ${ }_{2}$ agonist, as asthma maintenance therapy in multiple countries, including the USA and in the EU [10-12].

An integrated safety analysis and overview of efficacy data from Phase II and III studies, which assessed the overall benefit and risks of once-daily FF $100 \mu \mathrm{g}$ or $200 \mu \mathrm{g}$ for patients with varying severities of persistent asthma, was carried out using all available data.

\section{Methods}

\section{Clinical studies}

Fourteen clinical studies (six Phase II and eight Phase III) were conducted (FF asthma global clinical development programme): 10 assessed FF only and four investigated FF/vilanterol with a FF arm (Table 1 [13-26]). In 12 studies, FF was administered using the ELLIPTA ${ }^{\circ}$ dry powder inhaler in the evening. The remaining studies used Rotadisk Diskhaler ${ }^{\text {rm }}$ [13] or Diskus ${ }^{\mathrm{rm}} /$ Accuhaler $^{\mathrm{rm}}$ [14], in the morning or evening.

Across the 14 studies, inclusion criteria were as follows: patients $\geq 12$ years of age; a clinical history of asthma (in accordance with the definition of National Institutes of Health [27]); forced expiratory volume in one second $\left(\mathrm{FEV}_{1}\right), 40-90 \%$ of the predicted normal value $(50-90 \%$ in one study [24]); bronchodilator reversibility of disease $\left(\geq 12 \%\right.$ and $\geq 200 \mathrm{~mL}$ increase in $\mathrm{FEV}_{1}$ within $10-40 \mathrm{~min}$ following two to four inhalations of albuterol/salbutamol); and documented use of albuterol/salbutamol and/or asthma symptoms on $\geq 4$ of the last 7 consecutive days of a run-in period (or in one study, $\geq 3$ of the last 7 consecutive days of the run-in period [24]). As patients entering the Phase III studies were symptomatic on ICS or ICS/ LABA therapy, their asthma severity may be considered equivalent to GINA steps 3-4 [1].

All studies complied with the principles of Good Clinical Practice [28] and were approved by relevant Ethics Committees/Institutional Review Boards. Written informed consent was obtained. Studies were conducted in accordance with the applicable version of the Declaration of Helsinki [29]. Regulatory approval was obtained from the relevant health authority where required.

\section{Safety analysis}

Key comparisons of interest for integrated safety analyses were FF $100 \mu \mathrm{g}$ versus placebo and FF $200 \mu \mathrm{g}$ versus placebo. Data from 10 completed Phase II and III parallelgroup, double-blind studies, in which FF was delivered via the ELLIPTA ${ }^{\circ}$ inhaler, were integrated to assess FF safety (Table 1). The four additional studies provided further supporting safety data for FF [13-15, 26]; however, these were reviewed individually and not integrated as they had either a different design (e.g., crossover/open-label) or did not use the ELLIPTA ${ }^{\circ}$ inhaler. The treatment groups analysed were as follows: once-daily FF $50 \mu \mathrm{g}, 100 \mu \mathrm{g}$ and $200 \mu \mathrm{g}$; twice-daily fluticasone propionate (FP) $100 \mu \mathrm{g}$, $250 \mu \mathrm{g}$ and $500 \mu \mathrm{g}$; and placebo. All randomised patients who received at least one dose of study medication were included in the integrated analysis. As the data were integrated to support the regulatory filings of FF monotherapy for the treatment of asthma, the cut-off for this analysis was 15 February 2013; data from studies reported following this date were not included.

The integrated analysis determined (a) the rate of adverse events (AEs) and (b) the exposure-adjusted $\mathrm{AE}$ rate (to account for variation in treatment exposure across the groups) reported as the number of patients with an event per 1000 patient-years of exposure.

AEs were coded and grouped by System Organ Class and Preferred Term using the Medical Dictionary for Regulatory Activities (MedDRA, version 15.1). AEs of special interest (AESI) were defined using pre-selected MedDRA preferred terms and based on the known AE profile/pharmacology of corticosteroids: hypersensitivity, bone disorders, local steroid effects (e.g., oral candidiasis/ hoarseness), ocular effects, glucose effects, pneumonia, lower respiratory tract infection and systemic effects (e.g., hypothalamic-pituitary-adrenal [HPA] axis). A severe asthma exacerbation was defined as an asthma deterioration requiring the use of systemic or oral corticosteroids for $\geq 3$ days, or an in-patient hospitalisation or emergency department visit due to asthma that required systemic corticosteroids (asthma exacerbations were only recorded as AEs if they met the definition of a serious AE). Vital signs (diastolic and systolic blood pressure, heart rate) and laboratory assessments (clinical chemistry and haematology parameters) were also integrated.

The asthma clinical programme assessed 24-h urine and serum cortisol excretion. Urinary cortisol measurements were collected in seven of the 10 studies integrated for safety. Urinary cortisol excretion was log-transformed and analysed in the urine cortisol population (a subset of patients whose urine samples did not have confounding factors that could affect the interpretation of the results, e.g., inadequate urine volume, inappropriate collection time, 24-h creatinine excretion below the lower limit of threshold, use of prohibited medications or missing the 
Table 1 Clinical studies conducted as part of the FF global clinical development programme in asthma

\begin{tabular}{|c|c|c|c|c|c|c|c|}
\hline Study & Phase & $\begin{array}{l}\text { Number of } \\
\text { patients }\end{array}$ & Treatment arms & $\begin{array}{l}\text { Study duration, } \\
\text { weeks }\end{array}$ & Primary end-point & Additional end-points & $\begin{array}{l}\text { Included in integrated } \\
\text { safety analysis }\end{array}$ \\
\hline $\begin{array}{l}\text { Medley et al. [13] } \\
\text { (NCT01499446) }^{a}\end{array}$ & II dose regimen & 575 & $\begin{array}{l}\text { FF } 100 \mu \mathrm{g} \text { OD morning; } \\
\text { FF } 100 \mu \mathrm{g} \text { OD evening; } \\
\text { FF } 250 \mu \mathrm{g} \text { evening; placebo }\end{array}$ & 4 & $\begin{array}{l}\text { Mean change from baseline } \\
\text { in daily trough PEF }\end{array}$ & $\begin{array}{l}\mathrm{FEV}_{1} \text {, symptom-free days, } \\
\text { rescue-free days, withdrawals, } \\
\text { safety (AEs, 24-h UFC) }\end{array}$ & No \\
\hline $\begin{array}{l}\text { Woodcock et al. [14] } \\
\text { (NCT00398645) }\end{array}$ & Il dose regimen & 545 & $\begin{array}{l}\text { FF 200, } 400 \mu \mathrm{g} \text { OD morning; } \\
\text { FF 200, } 400 \mu \mathrm{g} \text { OD evening; } \\
\text { FF } 200 \mu \mathrm{g} \mathrm{BD;} \text { placebo }\end{array}$ & 8 & $\begin{array}{l}\text { Mean change from baseline } \\
\text { in trough } \mathrm{FEV}_{1}\end{array}$ & $\begin{array}{l}\text { Safety (AEs, withdrawals, 24-h } \\
\text { UFC) }\end{array}$ & No \\
\hline $\begin{array}{l}\text { Woodcock et al. [15] } \\
\text { (NCT00766090) }\end{array}$ & II dose regimen & 190 & $\begin{array}{l}\text { FF, FP } 200 \mu \mathrm{g} \text { OD evening; } \\
\text { FF, FP } 100 \mu \mathrm{g} \text { BD; placebo }\end{array}$ & 4 & $\begin{array}{l}\text { Mean change from baseline } \\
\text { in trough } \mathrm{FEV}_{1}\end{array}$ & Safety (AEs, 24-h UFC) & No \\
\hline $\begin{array}{l}\text { Busse et al. [16] }{ }^{c} \\
\text { (NCT00603746) }\end{array}$ & II dose ranging & 627 & $\begin{array}{l}\text { FF } 200,400,600,800 \mu \mathrm{g} \text { OD } \\
\text { evening; } \\
\text { FP } 500 \mu \mathrm{g} \text { BD; placebo }\end{array}$ & 8 & $\begin{array}{l}\text { Mean change from baseline } \\
\text { in trough } \mathrm{FEV}_{1}\end{array}$ & $\begin{array}{l}\text { Asthma symptom scores, PEF, } \\
\text { symptom-free days, rescue-free } \\
\text { days, withdrawals, safety (AEs, } \\
\text { 24-h UFC) }\end{array}$ & Yes \\
\hline $\begin{array}{l}\text { Bleecker et al. [17] } \\
\text { (NCT00603278) }\end{array}$ & II dose ranging & 622 & $\begin{array}{l}\text { FF 100, 200, 300, } 400 \mu \mathrm{g} \text { OD } \\
\text { evening; FP } 250 \mu \mathrm{gBD} \text {; } \\
\text { placebo }\end{array}$ & 8 & $\begin{array}{l}\text { Mean change from baseline } \\
\text { in trough } \mathrm{FEV}_{1}\end{array}$ & $\begin{array}{l}\text { PEF, symptom-free and rescue- } \\
\text { free periods, withdrawals, safety } \\
\text { (AEs, 24-h UFC) }\end{array}$ & Yes \\
\hline $\begin{array}{l}\text { Bateman et al. }[18]^{c} \\
\text { (NCT00603382) }\end{array}$ & II dose ranging & 598 & $\begin{array}{l}\text { FF } 25,50,100,200 \mu \mathrm{g} O D \\
\text { evening; } \\
\text { FP } 100 \mu \mathrm{g} \text { BD; placebo }\end{array}$ & 8 & $\begin{array}{l}\text { Mean change from baseline } \\
\text { in trough } \mathrm{FEV}_{1}\end{array}$ & $\begin{array}{l}\text { PEF, symptom-free and rescue- } \\
\text { free periods, withdrawals, safety } \\
\text { (AEs, 24-h UFC) }\end{array}$ & Yes \\
\hline $\begin{array}{l}\text { O'Byrne et al. [19] } \\
\text { (NCT01436071) }\end{array}$ & III efficacy & 248 & $\begin{array}{l}\text { FF } 50 \mu \mathrm{g} \text { OD evening; } \\
\text { placebo }\end{array}$ & 12 & $\begin{array}{l}\text { Mean change from baseline } \\
\text { in trough } \mathrm{FEV}_{1}\end{array}$ & $\begin{array}{l}\text { Rescue-free and symptom-free } \\
24-h \text { periods, PEF, ACT, QoL, } \\
\text { safety (AEs, severe exacerbations) }\end{array}$ & Yes \\
\hline $\begin{array}{l}\text { Busse et al. [20] }{ }^{c} \\
\text { (NCT01436110) }\end{array}$ & III efficacy & 351 & $\begin{array}{l}\text { FF } 50 \mu \mathrm{g} \text { OD evening; } \\
\text { FP } 100 \mu \mathrm{g} \mathrm{BD;} \\
\text { placebo }\end{array}$ & 24 & $\begin{array}{l}\text { Mean change from baseline } \\
\text { in trough evening } \mathrm{FEV}_{1}\end{array}$ & $\begin{array}{l}\text { Rescue-free and symptom-free } \\
24-h \text { periods, PEF, ACT, QoL, safety } \\
\text { (AEs, severe exacerbations) }\end{array}$ & Yes \\
\hline $\begin{array}{l}\text { Lötvall et al. [21] }{ }^{c} \\
\text { (NCT01159912) }\end{array}$ & III efficacy & 343 & $\begin{array}{l}\text { FF } 100 \mu \mathrm{g} \text { OD evening; } \\
\text { FP } 250 \text { mg BD; placebo }\end{array}$ & 24 & $\begin{array}{l}\text { Mean change from baseline } \\
\text { in trough evening } \mathrm{FEV}_{1}\end{array}$ & $\begin{array}{l}\text { Rescue-free and symptom-free } \\
\text { 24-h periods, PEF, ACT, QoL, } \\
\text { safety (AEs, severe exacerbations, } \\
\text { 24-h UFC) }\end{array}$ & Yes \\
\hline $\begin{array}{l}\text { Woodcock et al. [22] } \\
\text { (NCT01431950) }\end{array}$ & III efficacy & 238 & FF $100 \mu \mathrm{g}, 200 \mu \mathrm{g}$ OD evening & 24 & $\begin{array}{l}\text { Mean change from baseline } \\
\text { in trough } \mathrm{FEV}_{1}\end{array}$ & $\begin{array}{l}\text { Rescue-free and symptom-free } \\
24-h \text { periods, PEF, ACT, safety } \\
\text { (AEs, severe exacerbations, } \\
\text { 24-h UFC) }\end{array}$ & Yes \\
\hline $\begin{array}{l}\text { Bleecker et al. [23] } \\
\text { (NCT01165138) }\end{array}$ & III efficacy & 609 & $\begin{array}{l}\text { FF/ } 100 / 25 \mu \mathrm{g} \\
\text { FF } 100 \mu \mathrm{g} \text { OD evening; } \\
\text { placebo }\end{array}$ & 12 & $\begin{array}{l}\text { Mean change from baseline } \\
\text { in trough } \mathrm{FEV}_{1} \text { and serial } \\
(0-24 \mathrm{~h}) \text { weighted mean } \mathrm{FEV}_{1}\end{array}$ & $\begin{array}{l}\text { Rescue-free and symptom-free } \\
\text { 24-h periods, QoL, withdrawals, } \\
\text { safety (AEs, severe exacerbations, } \\
\text { 24-h UFC) }\end{array}$ & Yes \\
\hline $\begin{array}{l}\text { Bateman et al. [24] } \\
\text { (NCT01086384) }\end{array}$ & III efficacy & 2019 & $\begin{array}{l}\text { FFNI 100/25 } \mu \mathrm{g} \text {, FF } 100 \mu \mathrm{g} \text { OD } \\
\text { evening }\end{array}$ & $\geq 24-78$ & Time to first severe exacerbation & $\begin{array}{l}\text { Rate of severe exacerbations per } \\
\text { patient per year, trough } \mathrm{FEV}_{1} \text {, } \\
\text { safety (hospitalisations, AEs) }\end{array}$ & Yes \\
\hline
\end{tabular}


Table 1 Clinical studies conducted as part of the FF global clinical development programme in asthma (Continued)

\begin{tabular}{|c|c|c|c|c|c|c|c|}
\hline $\begin{array}{l}\text { O'Byrne et al. [25] } \\
\text { (NCT01134042) }^{c}\end{array}$ & III efficacy & 586 & $\begin{array}{l}\text { FF/NI 200/25 } \mu \mathrm{g} \text { OD evening; } \\
\text { FF } 200 \mu \mathrm{g} \text { OD evening; } \\
\text { FP } 500 \mu \mathrm{g} \mathrm{BD}\end{array}$ & 24 & $\begin{array}{l}\text { Mean change from baseline } \\
\text { in trough FEV }{ }_{1} \text { and serial } \\
\left(0-24 \text { h) weighted mean } \mathrm{FEV}_{1}\right.\end{array}$ & $\begin{array}{l}\text { Rescue-free } 24-h \text { and symptom- } \\
\text { free } 24-h \text { periods, QoL, PEF, ACT, } \\
\text { safety (AEs, 24-h UFC) }\end{array}$ & Yes \\
\hline $\begin{array}{l}\text { Muraki et al. [26] } \\
\text { (NCT01244984) }\end{array}$ & III safety & 243 & $\begin{array}{l}\text { FFNI 100/25 } \mu \mathrm{g}, \mathrm{FF} / \mathrm{I} \\
200 / 25 \mu \mathrm{g}, \mathrm{FF} 100 \mu \mathrm{g} \\
\text { OD evening }\end{array}$ & 52 & $\begin{array}{l}\text { Safety (AEs, severe exacerbations, } \\
\text { 24-h UFC) }\end{array}$ & PEF, asthma symptom scores & No \\
\hline
\end{tabular}

Clinicaltrials.gov study registration numbers are provided in brackets after each study citation

FF/VI 100/25 $\mu \mathrm{g}=92 / 22 \mu \mathrm{g}$ (emitted). FF/NI 200/25 $\mu \mathrm{g}=184 / 22 \mu \mathrm{g}$ (emitted). FF $100 \mu \mathrm{g}=90 \mu \mathrm{g}$ (emitted). FF $200 \mu \mathrm{g}=182 \mu \mathrm{g}$ (emitted)

$A C T$ asthma control test ${ }^{T M}, A E$ adverse event, $B D$ twice daily, $F E V$, forced expiratory volume in one second, $F F$ fluticasone furoate, $F P$ fluticasone propionate, $O D$ once daily, $P E F$ peak expiratory flow, $Q O L$ quality of life,

UFC urinary free cortisol excretion, $V I$ vilanterol

${ }^{\mathrm{a}} \mathrm{FF}$ administered via Rotadisk Diskhaler ${ }^{\mathrm{TM}}$

${ }^{\mathrm{b} F F}$ administered via Diskus ${ }^{\mathrm{TM}} /$ Accuhaler $^{\mathrm{TM}}$

${ }^{\mathrm{C} F F}$ administered via ELLIPTA ${ }^{\circ}$ inhaler 
baseline and/or end of treatment assessments). An analysis of covariance model was used, controlling for the following baseline effects (log): region, sex, age, treatment and study.

\section{Efficacy analysis}

Three strengths of FF monotherapy were assessed as part of a Phase III programme [19-26]: FF $50 \mu \mathrm{g}, 100 \mu \mathrm{g}$ and $200 \mu \mathrm{g}$ (Table 1). The efficacy of FF $50 \mu \mathrm{g}$ was not demonstrated and was not submitted for regulatory approval. The efficacy assessment mainly used data from individual studies. Trough (24-h post-dose for once-daily FF) $\mathrm{FEV}_{1}$, serial 0-24-h weighted mean $\mathrm{FEV}_{1}$ and peak expiratory flow (PEF) were evaluated, as well as symptomatic end-points (rescue-free and symptom-free 24-h periods) and Asthma Control Test ${ }^{\mathrm{rm}}$ score. To support regulatory submissions, the only two Phase III studies that were placebo controlled and included FF $100 \mu \mathrm{g}$ were integrated.

Access to the datasets supporting the conclusions of this manuscript may be obtained via https://www. clinicalstudydatarequest.com/.

\section{Results}

A total of 4203 patients in the 10 studies that were integrated for the safety analysis received at least one dose of study drug (3345 patients received at least one dose of FF). Treatment exposure varied across treatment groups from 60.28 to 1179.36 patient-years, with the greatest exposure reported for the $\mathrm{FF} 100 \mu \mathrm{g}$ group (Additional file 1: Table S1). As a placebo comparator is not ethical in longer-term studies, a placebo arm was not included in studies longer than 24 weeks. A total of 537 patients (32\%) in the FF $100 \mu \mathrm{g}$ group were treated for $>52$ weeks.

\section{Safety}

A summary of the $\mathrm{AE}$ incidence and exposure-adjusted $\mathrm{AE}$ incidence for each treatment arm is provided (Table 2). The most frequently reported AEs for the FF treatment groups in the integrated analysis were headache, nasopharyngitis, upper respiratory tract infection, bronchitis, oropharyngeal pain and cough (Table 3). There were no additional findings in the non-integrated studies.

Nasopharyngitis, oropharyngeal pain, sinusitis, influenza, back pain, dysphonia and viral respiratory tract infection occurred at a higher rate in patients treated with FF $100 \mu \mathrm{g}$ and $200 \mu \mathrm{g}$, versus placebo. However, there were generally no differences in the exposure-adjusted $\mathrm{AE}$ rates for $\mathrm{FF}$ $100 \mu \mathrm{g}$ versus placebo, except for back pain and viral respiratory tract infection. Similarly, there were no clear differences in exposure-adjusted AE rates between FF and FP.

In two long-term studies (FF $100 \mu \mathrm{g}$ for $>6$ months $[24,26])$, there was generally no difference in the pattern of new $\mathrm{AE}$ occurrence after 6 months and no increased incidence of AEs during the second 6 months, compared with the first 6 months. For example, in one study of up to 78 weeks, the $\mathrm{AE}$ incidence with an onset in the first 6 months was $54 \%$, compared with $38 \%$ with an onset of after 6 months. There was no pattern to suggest a difference in the $\mathrm{AE}$ profile according to length of study medication exposure.

The most frequently reported drug-related AEs in the FF treatment groups were headache, dysphonia and oral/ oropharyngeal candidiasis. The incidence of drug-related AEs ranged from $2 \%$ with FF $50 \mu$ g to $6 \%$ with FF $100 \mu \mathrm{g}$ and $200 \mu \mathrm{g}$ for once-daily administration, and up to $8 \%$ with twice-daily FP $250 \mu \mathrm{g}$ and FP $500 \mu \mathrm{g}$.

Serious AEs (SAEs) were experienced by $<1-2 \%$ of patients across the treatment groups (Table 2), most frequently in the FF $100 \mu \mathrm{g}$ group $(2 \%, n=38)$. Asthma exacerbation was the most common SAE, experienced by nine patients in the FF $100 \mu \mathrm{g}$ group and one patient in each of the placebo, FF $200 \mu \mathrm{g}$ and twice-daily FP $500 \mu \mathrm{g}$ groups. All nine patients with severe asthma exacerbations in the FF $100 \mu \mathrm{g}$ groups were from a study that specifically examined exacerbations. That study was $\leq 78$ weeks in duration (compared with $\leq 24$ weeks in other studies), had no placebo arm and, unlike other studies, recruited patients with a history of severe asthma exacerbations in the previous year [24]. Only four SAEs

Table 2 Summary of the AE profile for the integrated clinical studies

\begin{tabular}{|c|c|c|c|c|c|c|c|}
\hline AE (preferred term), $n(\%)$ & Placebo $(n=858)$ & $\begin{array}{l}\text { FF } 50 \mu \mathrm{g} \text { OD } \\
(n=338)\end{array}$ & $\begin{array}{l}\text { FF } 100 \mu \mathrm{g} \text { OD } \\
(n=1663)\end{array}$ & $\begin{array}{l}\text { FF } 200 \mu \mathrm{g} \text { OD } \\
(n=608)\end{array}$ & $\begin{array}{l}\text { FP } 100 \mu \mathrm{g} \mathrm{BD} \\
(n=217)\end{array}$ & $\begin{array}{l}\text { FP } 250 \mu g \text { BD } \\
(n=214)\end{array}$ & $\begin{array}{l}\text { FP } 500 \mu \mathrm{g} \mathrm{BD} \\
(n=305)\end{array}$ \\
\hline Any AE & $278(32)$ & $121(36)$ & $912(55)$ & $256(42)$ & $94(43)$ & $90(42)$ & $136(45)$ \\
\hline Any drug-related $\mathrm{AE}$ & $22(3)$ & $8(2)$ & $103(6)$ & $36(6)$ & $10(5)$ & $18(8)$ & $24(8)$ \\
\hline Any SAE & $7(<1)$ & $1(<1)$ & $38(2)$ & $7(1)$ & $3(1)$ & $1(<1)$ & $4(1)$ \\
\hline Any drug-related SAE & 0 & 0 & $3(<1)$ & 0 & 0 & 0 & $1(<1)$ \\
\hline $\begin{array}{l}\text { Any AE leading to } \\
\text { discontinuation of } \\
\text { study drug }\end{array}$ & $8(<1)$ & $2(<1)$ & $28(2)$ & $10(2)$ & $4(2)$ & $4(2)$ & $6(2)$ \\
\hline Deaths & 0 & 0 & $2(<1)^{\mathrm{a}}$ & 0 & 0 & 0 & 0 \\
\hline
\end{tabular}

$A E$ adverse event, $B D$ twice daily, $F F$ fluticasone furoate, $O D$ once daily, $F P$ fluticasone propionate, $S A E$ serious adverse event

${ }^{a}$ Neither death was determined by the investigator to be related to study medication 
Table 3 Most frequent on-treatment AEs reported with $\geq 3 \%$ incidence in any treatment groups (integrated clinical studies)

\begin{tabular}{|c|c|c|c|c|c|c|c|}
\hline AE (preferred term), $n^{a}(\%)$ & $\begin{array}{l}\text { Placebo } \\
(n=858)\end{array}$ & $\begin{array}{l}\text { FF } 50 \mu \mathrm{g} \text { OD } \\
(n=338)\end{array}$ & $\begin{array}{l}\text { FF } 100 \mu \mathrm{g} \text { OD } \\
(n=1663)\end{array}$ & $\begin{array}{l}\text { FF } 200 \mu \mathrm{g} \text { OD } \\
(n=608)\end{array}$ & $\begin{array}{l}\mathrm{FP} 100 \mu \mathrm{g} \mathrm{BD} \\
(n=217)\end{array}$ & $\begin{array}{l}\mathrm{FP} 250 \mu \mathrm{g} \mathrm{BD} \\
(n=214)\end{array}$ & $\begin{array}{l}\mathrm{FP} 500 \mu \mathrm{g} \mathrm{BD} \\
(n=305)\end{array}$ \\
\hline Any AE & $278(32)$ & $121(36)$ & $912(55)$ & $256(42)$ & $94(43)$ & $90(42)$ & $136(45)$ \\
\hline Headache & $66(8)$ & $29(9)$ & $228(14)$ & $44(7)$ & $24(11)$ & $15(7)$ & $25(8)$ \\
\hline Nasopharyngitis & $45(5)$ & $15(4)$ & $181(11)$ & $53(9)$ & $14(6)$ & $11(5)$ & $43(14)$ \\
\hline URTI & $16(2)$ & $8(2)$ & $111(7)$ & $15(2)$ & $7(3)$ & $12(6)$ & $7(2)$ \\
\hline Bronchitis & $15(2)$ & 0 & $98(6)$ & $15(2)$ & $3(1)$ & $5(2)$ & $7(2)$ \\
\hline Oropharyngeal pain & $11(1)$ & $2(<1)$ & $71(4)$ & $19(3)$ & $4(2)$ & $6(3)$ & $11(4)$ \\
\hline Cough & $9(1)$ & $3(<1)$ & $68(4)$ & $13(2)$ & $2(<1)$ & $5(2)$ & $15(5)$ \\
\hline Pharyngitis & $24(3)$ & $14(4)$ & $55(3)$ & $8(1)$ & $5(2)$ & $2(<1)$ & $7(2)$ \\
\hline Sinusitis & $8(<1)$ & $5(1)$ & $53(3)$ & $15(2)$ & $6(3)$ & $5(2)$ & $6(2)$ \\
\hline Influenza & $9(1)$ & $5(1)$ & $45(3)$ & $17(3)$ & $6(3)$ & 0 & $7(2)$ \\
\hline Back pain & $4(<1)$ & $7(2)$ & $52(3)$ & $11(2)$ & $4(2)$ & $2(<1)$ & $4(1)$ \\
\hline Dysphonia & $4(<1)$ & $1(<1)$ & $23(1)$ & $11(2)$ & $3(1)$ & $6(3)$ & $6(2)$ \\
\hline Rhinitis & $7(<1)$ & $1(<1)$ & $27(2)$ & $7(1)$ & $3(1)$ & 0 & $8(3)$ \\
\hline \multirow[t]{2}{*}{ Viral respiratory tract infection } & 0 & $2(<1)$ & $18(1)$ & $8(1)$ & 0 & 0 & $8(3)$ \\
\hline & \multicolumn{7}{|c|}{ Exposure-adjusted incidence rate per 1000 patient-years } \\
\hline $\begin{array}{l}\mathrm{AE} \\
\text { (preferred term), } n(\%)\end{array}$ & Placebo & FF $50 \mu \mathrm{g} O D$ & FF $100 \mu \mathrm{g} O D$ & FF $200 \mu \mathrm{g} O D$ & FP $100 \mu \mathrm{g} \mathrm{BD}$ & FP $250 \mu \mathrm{g} \mathrm{BD}$ & $\mathrm{FP} 500 \mu \mathrm{g}$ BD \\
\hline Patient-years & 185.6 & 87.5 & 1179.4 & 169.2 & 61.0 & 60.3 & 95.7 \\
\hline Headache & 355.6 & 331.5 & 193.3 & 260.1 & 393.4 & 248.8 & 261.2 \\
\hline Nasopharyngitis & 242.5 & 171.5 & 153.5 & 313.3 & 229.5 & 182.5 & 449.3 \\
\hline URTI & 86.2 & 91.4 & 94.1 & 88.7 & 114.8 & 199.1 & 73.1 \\
\hline Bronchitis & 80.8 & 0 & 83.1 & 88.7 & 49.2 & 82.9 & 73.1 \\
\hline Oropharyngeal pain & 59.3 & 22.9 & 60.2 & 112.3 & 65.6 & 99.5 & 114.9 \\
\hline Cough & 48.5 & 34.3 & 57.7 & 76.9 & 32.8 & 82.9 & 156.7 \\
\hline Pharyngitis & 129.3 & 160.0 & 46.6 & 47.3 & 82.0 & 33.2 & 73.1 \\
\hline Sinusitis & 43.1 & 57.2 & 44.9 & 88.7 & 98.4 & 82.9 & 62.7 \\
\hline Influenza & 48.5 & 57.2 & 38.2 & 100.5 & 98.4 & 0 & 73.1 \\
\hline Back pain & 21.6 & 80.0 & 44.1 & 65.0 & 65.6 & 33.2 & 41.8 \\
\hline Dysphonia & 21.6 & 11.4 & 19.5 & 65.0 & 49.2 & 99.5 & 62.7 \\
\hline Rhinitis & 37.7 & 11.4 & 22.9 & 41.4 & 49.2 & 0 & 83.6 \\
\hline Viral respiratory tract infection & 0 & 22.9 & 15.3 & 47.3 & 0 & 0 & 83.6 \\
\hline
\end{tabular}

$A E$ adverse event, $B D$ twice daily, $F F$ fluticasone furoate, $F P$ fluticasone propionate, $O D$ once daily, URTI upper respiratory tract infection

${ }^{a}$ Numbers represent the number of patients with an event per 1000 patient-years of exposure

were considered by the investigator to be treatment related: pneumonia, asthma exacerbation and non-cardiac chest pain with FF $100 \mu \mathrm{g}$, and haemoptysis with twicedaily FP $500 \mu \mathrm{g}$. There was no notable difference in the incidence of AEs leading to withdrawal across treatment groups $(0-2 \%$; Table 2); the most frequent of these were asthma exacerbation, dyspnoea and pneumonia.

Local steroid effects (comprising oropharyngeal pain, dysphonia, oral candidiasis and oropharyngeal candidiasis) were the most frequent AESI; the exposure-adjusted incidence per 1000 patient-years was 80.8 (placebo), 103.4 (FF $100 \mu \mathrm{g}$ ) and 283.8 (FF $200 \mu \mathrm{g}$; Table 4). No AEs indicative of HPA axis disorders were reported. The pneumonia incidence was low $(\leq 0.7 \%)$ across all groups (Additional file 2: Figure S1). The exposure-adjusted incidence of pneumonia (Table 4) was similar between FF $100 \mu \mathrm{g}$ and placebo, and was numerically higher with FF $200 \mu \mathrm{g}(8.5,10.8$ and 23.6, respectively, per 1000 patientyears); however, the confidence intervals (CIs) were wide and overlapped across all groups, including placebo (Additional file 2: Figure S1). Serious pneumonia occurred at comparable rates for FF $100 \mu \mathrm{g}$, FF $200 \mu \mathrm{g}$ and placebo (4.2, 5.9 and 5.4, respectively, per 1000 patient-years; Additional file 3: Figure S2).

Urinary cortisol levels were measured in seven of the 10 integrated studies (Table 1). At the end of 
Table 4 AESI occurring in any treatment groups (integrated clinical studies)

\begin{tabular}{|c|c|c|c|c|c|c|c|}
\hline AE of special interest (preferred term), $n^{\mathrm{a}}(\%)$ & $\begin{array}{l}\text { Placebo } \\
(n=858)\end{array}$ & $\begin{array}{l}\text { FF } 50 \mu \mathrm{g} O D \\
(n=338)\end{array}$ & $\begin{array}{l}\mathrm{FF} 100 \mu \mathrm{g} \text { OD } \\
(n=1663)\end{array}$ & $\begin{array}{l}\mathrm{FF} 200 \mu \mathrm{g} \text { OD } \\
(n=608)\end{array}$ & $\begin{array}{l}\mathrm{FP} 100 \mu \mathrm{g} \mathrm{BD} \\
(n=217)\end{array}$ & $\begin{array}{l}\mathrm{FP} 250 \mu \mathrm{g} \mathrm{BD} \\
(n=214)\end{array}$ & $\begin{array}{l}\mathrm{FP} 500 \mu \mathrm{g} \mathrm{BD} \\
(n=305)\end{array}$ \\
\hline Local steroid effects & $15(2)$ & $7(2)$ & $122(7)$ & $48(8)$ & $8(4)$ & $18(8)$ & $25(8)$ \\
\hline Oropharyngeal pain & $11(1)$ & $2(<1)$ & $71(4)$ & $19(3)$ & $4(2)$ & $6(3)$ & $11(4)$ \\
\hline Dysphonia & $4(<1)$ & $1(<1)$ & $23(1)$ & $11(2)$ & $3(1)$ & $6(3)$ & $6(2)$ \\
\hline Oral candidiasis & 0 & $4(1)$ & $18(1)$ & $8(1)$ & $1(<1)$ & $4(2)$ & $4(1)$ \\
\hline Oropharyngeal candidiasis & $1(<1)$ & $1(<1)$ & $7(<1)$ & $9(1)$ & $1(<1)$ & $2(<1)$ & $6(2)$ \\
\hline LRTI excluding pneumonia & $16(2)$ & $1(<1)$ & $114(7)$ & $19(3)$ & $3(1)$ & $5(2)$ & $7(2)$ \\
\hline Bronchitis & $15(2)$ & 0 & $98(6)$ & $15(2)$ & $3(1)$ & $5(2)$ & $7(2)$ \\
\hline Hypersensitivity ${ }^{b}$ & $13(2)$ & $3(<1)$ & $41(2)$ & $6(<1)$ & $2(<1)$ & $2(<1)$ & $6(2)$ \\
\hline Bone disorders ${ }^{\mathrm{b}}$ & 0 & $2(<1)$ & $21(1)$ & $2(<1)$ & $1(<1)$ & 0 & $4(1)$ \\
\hline Pneumonia ${ }^{\mathrm{b}}$ & $2(<1)$ & 0 & $10(<1)$ & $4(<1)$ & $1(<1)$ & 0 & 0 \\
\hline Effects on glucose ${ }^{b}$ & 0 & 0 & $11(<1)$ & $2(<1)$ & 0 & 0 & 0 \\
\hline \multirow[t]{2}{*}{ Ocular effects ${ }^{b}$} & 0 & 0 & $6(<1)$ & 0 & 0 & 0 & 0 \\
\hline & & \multicolumn{6}{|c|}{ Exposure-adjusted incidence rate per 1000 patient-years } \\
\hline AE of special interest (preferred term), $n(\%)$ & Placebo & FF $50 \mu \mathrm{g} O D$ & FF $100 \mu \mathrm{g}$ OD & FF $200 \mu \mathrm{g}$ OD & FP $100 \mu \mathrm{g} B D$ & FP $250 \mu \mathrm{g} \mathrm{BD}$ & FP $500 \mu \mathrm{g} B D$ \\
\hline Patient-years & 185.6 & 87.5 & 1179.4 & 169.2 & 61.0 & 60.3 & 95.7 \\
\hline Local steroid effects & 80.8 & 80.0 & 103.4 & 283.8 & 131.1 & 298.6 & 261.2 \\
\hline Oropharyngeal pain & 59.3 & 22.9 & 60.2 & 112.3 & 65.6 & 99.5 & 114.9 \\
\hline Dysphonia & 21.6 & 11.4 & 19.5 & 65.0 & 49.2 & 99.5 & 62.7 \\
\hline Oral candidiasis & 0 & 45.7 & 15.3 & 47.3 & 16.4 & 66.4 & 41.8 \\
\hline Oropharyngeal candidiasis & 5.4 & 11.4 & 5.9 & 53.2 & 16.4 & 33.2 & 62.7 \\
\hline LRTI excluding pneumonia & 86.2 & 11.4 & 96.7 & 112.3 & 49.2 & 82.9 & 73.1 \\
\hline Bronchitis & 80.8 & 0 & 83.1 & 88.7 & 49.2 & 82.9 & 73.1 \\
\hline Hypersensitivity ${ }^{\mathrm{b}}$ & 70.0 & 34.3 & 34.8 & 35.5 & 32.8 & 33.2 & 62.7 \\
\hline Bone disorders ${ }^{\mathrm{b}}$ & 0 & 22.9 & 17.8 & 11.8 & 16.4 & 0 & 41.8 \\
\hline Pneumonia ${ }^{b}$ & 10.8 & 0 & 8.5 & 23.6 & 16.4 & 0 & 0 \\
\hline Effects on glucose $\mathrm{e}^{\mathrm{b}}$ & 0 & 0 & 9.3 & 11.8 & 0 & 0 & 0 \\
\hline Ocular effects ${ }^{\mathrm{b}}$ & 0 & 0 & 5.1 & 0 & 0 & 0 & 0 \\
\hline
\end{tabular}

$A E$ adverse event, $B D$ twice daily, $F F$ fluticasone furoate, $F P$ fluticasone propionate, $L R T /$ lower respiratory tract infection, $O D$ once daily

${ }^{a}$ Numbers represent the number of patients with an event per 1000 patient-years of exposure

${ }^{\mathrm{b}}$ No individual event occurred in $\geq 1 \%$ of patients

treatment, 24-h mean urinary cortisol excretion levels were similar to baseline levels and all mean ratios from treatment end to baseline were close to 1 in the oncedaily FF $100 \mu \mathrm{g}$ and $200 \mu \mathrm{g}$, twice-daily FP $100 \mu \mathrm{g}, 250 \mu \mathrm{g}$ and $500 \mu \mathrm{g}$, and placebo groups. In the adjusted means analysis, there were no statistically significant differences in 24-h urinary cortisol excretion from baseline to treatment end between each FF treatment group and placebo (Fig. 1); the percentage of patients with a change from normal urinary cortisol levels at baseline to low levels during treatment was similar between the FF $(<1 \%$, FF $100 \mu \mathrm{g}$, and 3\%, FF $200 \mu \mathrm{g}$ ) and placebo (2\%) treatment groups.

Vital sign and laboratory assessments were reviewed as part of the integrated analysis; no apparent treatmentrelated effects were observed.

\section{Efficacy in Phase II/III studies}

\section{Lung function}

Across the Phase II and III studies, FF $100 \mu \mathrm{g}$ and $200 \mu \mathrm{g}$ consistently improved trough $\mathrm{FEV}_{1}$, compared with placebo (Fig. 2). In Phase III studies, the treatment differences between FF $100 \mu \mathrm{g}$ and placebo for change from baseline in trough $\mathrm{FEV}_{1}$ were $146 \mathrm{~mL}$ (95\% CI: 36257 [21]) and $136 \mathrm{~mL}$ (95\% CI: 51-222 [23]), at 24 weeks and 12 weeks, respectively. In one study, weighted mean $\mathrm{FEV}_{1}$ (0-24 h; change from baseline) was measured and FF $100 \mu \mathrm{g}$ demonstrated a statistically significant difference of $186 \mathrm{~mL}$ (95\% CI: 62-310) from placebo at the end of the 12-week treatment period [23]. In another study, the efficacy of once-daily FF $200 \mu \mathrm{g}$ was similar to twice-daily FP $500 \mu \mathrm{g}$ in terms of trough and 0-24-h weighted mean $\mathrm{FEV}_{1}$ improvement [25]. Finally, in a 


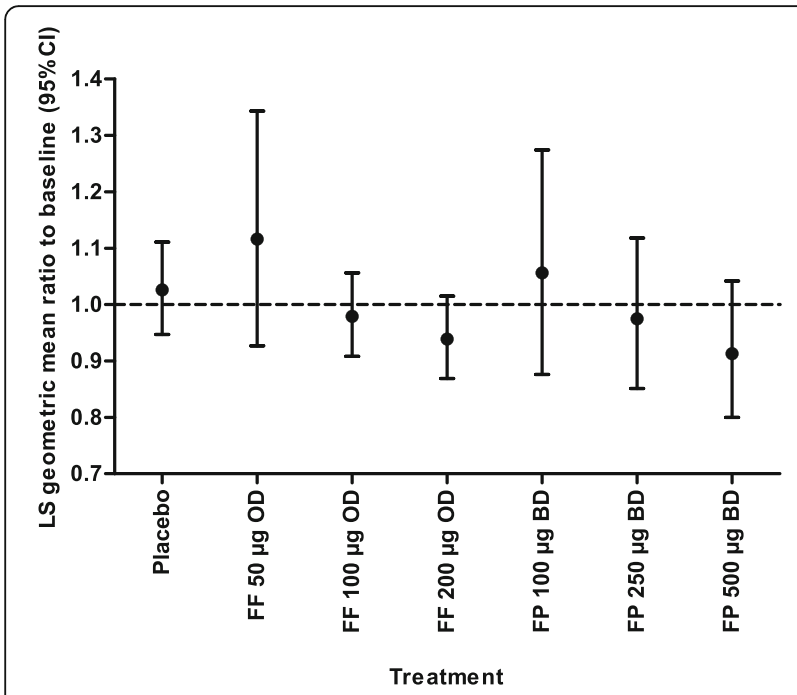

Fig. 1 Least squares geometric mean ratio to baseline (95\% Cl) in urinary free cortisol excretion at end of treatment (integrated clinical studies, urine cortisol population). Analysis performed using ANCOVA with covariates of region, study, gender, age, treatment and the log of the baseline values. Includes studies NCT00603746, NCT00603278, NCT00603382, NCT01159912, NCT01431950, NCT01 165138 and NCT01134042 [16-18, 21-23, 25]. Abbreviations: ANCOVA, analysis of covariance; $B D$, twice daily; $C l$, confidence interval; $F F$, fluticasone furoate; $F P$, fluticasone propionate; $L S$, least squares; $O D$, once daily
Phase II study, patients with persistent asthma not controlled by short-acting beta ${ }_{2}$ agonists had significant $\mathrm{FEV}_{1}$ and evening PEF improvements after receiving FF $100 \mu \mathrm{g}$ or $200 \mu$ g, versus placebo $(p \leq 0.005$ [18]).

\section{Symptomatic end-points}

FF $100 \mu \mathrm{g}$ and $200 \mu \mathrm{g}$ resulted in improvements in the proportion of rescue-free 24 -h periods versus placebo in all studies where this was measured (Fig. 3). In the only two Phase III studies that compared FF with placebo, FF $100 \mu \mathrm{g}$ demonstrated significant improvements in the proportion of rescue-free $24-\mathrm{h}$ periods versus placebo $(14.8 \%, p<0.001$ [23], and $8.7 \%, p=0.007$ [21]).

\section{Exacerbations}

Exacerbations were assessed in the integrated safety analysis. Patients who received FF $100 \mu \mathrm{g}$ had a 53.4\% risk reduction for a severe asthma exacerbation by the end of the treatment period, compared with placebo (hazard ratio [HR]: 0.466, 95\% CI: $0.240-0.906 ; p=0.024)$. Relative to placebo-treated patients, risk reductions for severe asthma exacerbations were also seen with FF $200 \mu \mathrm{g}$ (64.8\% risk reduction; HR: 0.352 , 95\% CI: $0.171-0.724 ; p=0.005$ ).

\section{Discussion}

In this integrated analysis, FF had an acceptable safety profile, comparable with other ICS. The specific effects

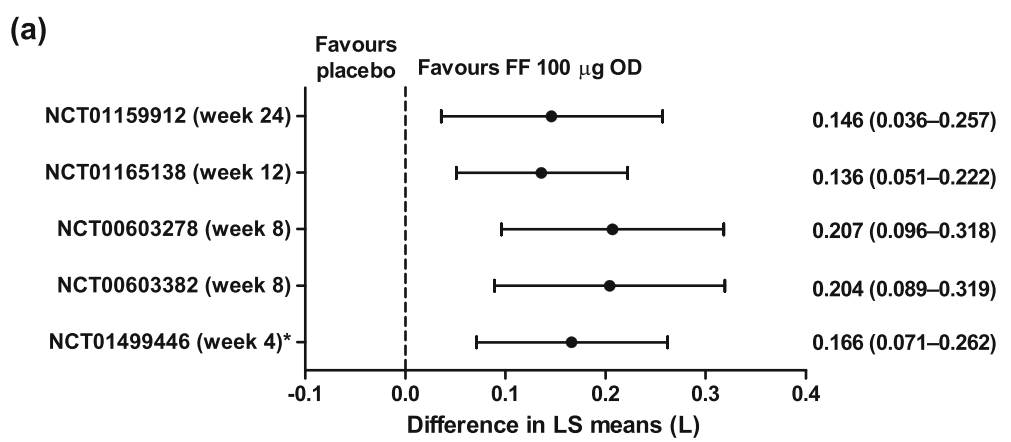

(b)

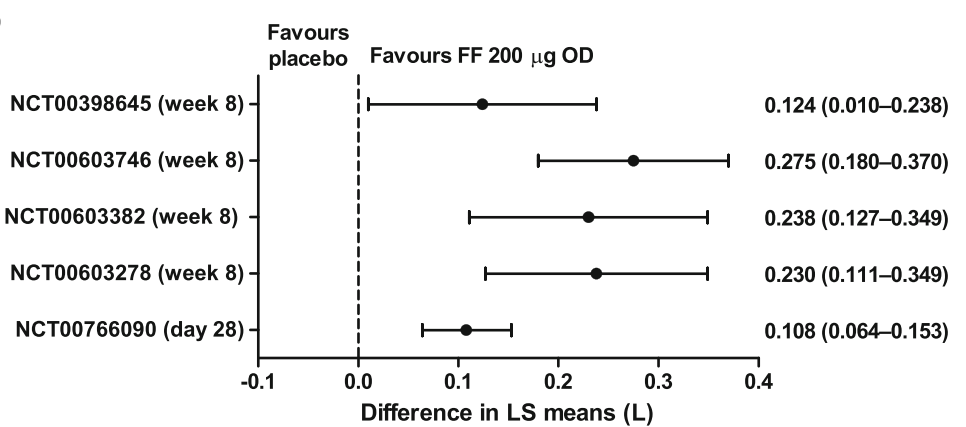

Fig. 2 Forest plots for change from baseline in trough FEV . (a) FF $100 \mu \mathrm{g}$ versus placebo (ITT population), and (b) FF $200 \mu \mathrm{g}$ versus placebo (ITT population). *In study NCT01499446, the final approved inhaler for FF delivery was not used [13]. Abbreviations: FEV ${ }_{1}$, forced expiratory volume in one second; $F F$, fluticasone furoate; ITT, intent-to-treat; $L S$, least squares; $O D$, once daily 

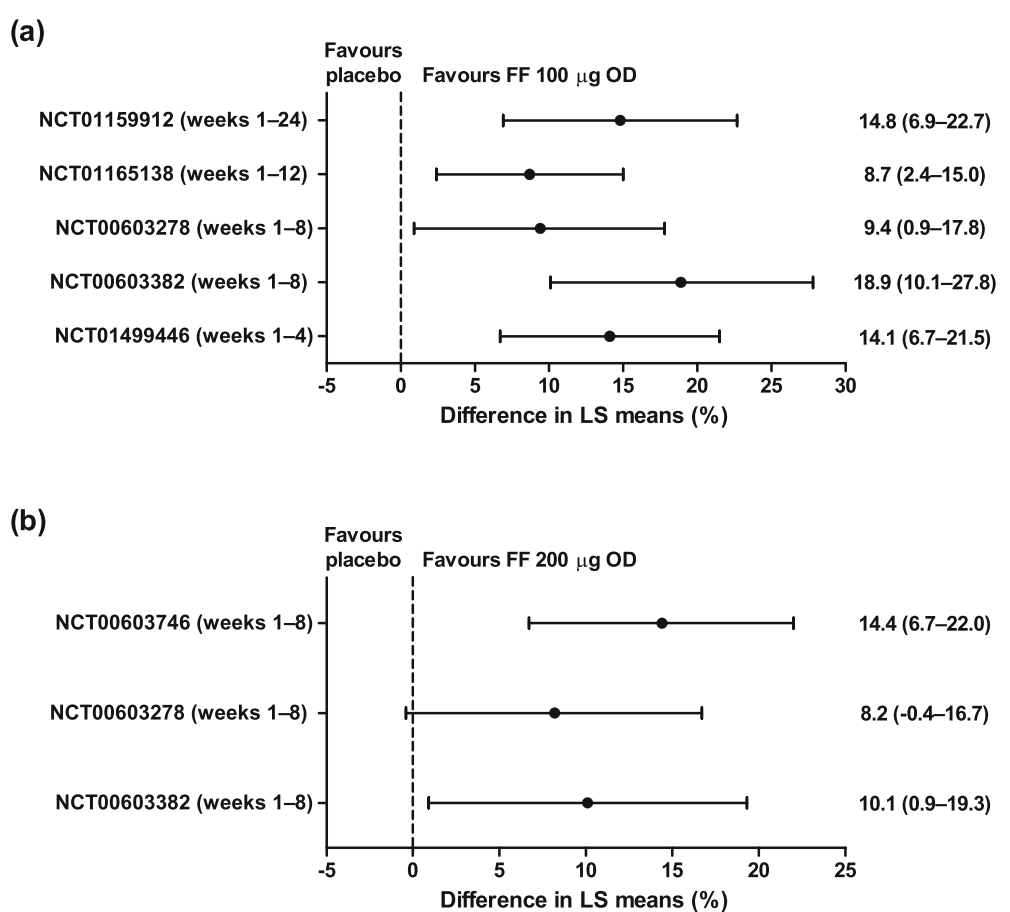

Fig. 3 Forest plots for change from baseline $(95 \% \mathrm{Cl}$ ) in percentage of rescue-free $24-\mathrm{h}$ periods. (a) FF $100 \mu \mathrm{g}$ versus placebo (ITT population), and (b) FF $200 \mathrm{\mu g}$ versus placebo (ITT population). Abbreviations: Cl, confidence interval; $F F$, fluticasone furoate; ITT, intent-to-treat; $L S$, least squares; $O D$, once daily

seen were consistent with known ICS class effects. The most frequently reported AEs were as anticipated, having been reported with other ICS (upper respiratory tract infection, bronchitis, oropharyngeal pain and cough [30]) or commonly experienced by patients with asthma (headache and nasopharyngitis were reported at similar frequencies in the placebo group). Despite a dose-response relationship between ICS use in asthma and oral candidiasis being well recognised, oral/oropharyngeal candidiasis rates were low and did not occur in more than $2 \%$ of FF- or FPtreated patients in this integrated analysis. However, the exposure-adjusted presentation of AESI indicated a higher incidence of local steroid effects for FF $200 \mu \mathrm{g}$, compared with FF $100 \mu \mathrm{g}$ and placebo.

Pneumonia was pre-specified as an AESI because it has been reported as an ICS-related event in chronic obstructive pulmonary disease, but no clear association has been identified with the use of ICS and pneumonia in asthma [31]. When assessing AEs across multiple studies of varying lengths and population size, it is important to consider exposure-adjusted incidence to account for disparities. In this integrated analysis, the incidence of pneumonia was low $(<1 \%)$ in all treatment groups and, although the exposure-adjusted incidence of pneumonia was slightly higher with FF $200 \mu \mathrm{g}$ than FF $100 \mu \mathrm{g}$ or placebo, the CIs were wide and overlapped (Additional file 2: Figure S1). An increased pneumonia incidence with higher doses cannot be ruled out, but the absolute risk of pneumonia was small and the exposure-adjusted incidence rates were consistent with the background rate in the asthmatic population [32] and, for FF $200 \mu \mathrm{g}$, comparable to that calculated for placebo, FP and budesonide in a meta-analysis [31]. There was no evidence of a higher rate of other AESI (hypersensitivity, bone disorders, adrenal suppression, ocular effects and effects on glucose [based on known pharmacological effects of corticosteroids]), relative to placebo or active comparator.

Beyond the present analysis, the safety profile of FF is supported by the clinical experience of FF in combination with vilanterol. Long-term safety evaluation of $\mathrm{FF} /$ vilanterol 100/25 $\mu$ g and FF/vilanterol 200/25 $\mu$ g has not identified any additional potential corticosteroid-related effects or significant findings from formal ophthalmological examinations after 52 weeks of exposure [33].

Cortisol suppression has been observed in patients with asthma who have normal HPA axis function at baseline receiving high doses of ICS [34]. However, in the studies included in our analyses there were no reports of significant cortisol suppression. In a formal HPA axis study, measurement of 24-h serum cortisol (a sensitive method for assessing adrenocortical activity) was used to assess non-inferiority of FF/vilanterol (100/25 $\mu \mathrm{g}$ and 200/25 $\mu \mathrm{g})$ compared with placebo [35]. There were no statistically significant differences in $0-24$-h weighted mean serum 
cortisol between either FF/vilanterol treatment and placebo after 6 weeks of treatment, but the active control (7 days of once-daily prednisolone $10 \mathrm{mg}$ ) significantly reduced serum cortisol levels. In the present study, few patients in any treatment group experienced below-normal levels of urinary free cortisol excretion at the end of treatment, and the incidence reported within the FF groups was similar to that in placebo.

In the efficacy analysis, once-daily FF $100 \mu \mathrm{g}$ and $200 \mu \mathrm{g}$ treatment for patients with persistent asthma produced consistent trough $\mathrm{FEV}_{1}$ improvements and increases in the proportion of rescue-free 24-h periods, compared with placebo. This present analysis examined evening dosing (primarily), but a subsequent study has suggested that morning and evening FF doses are equally efficacious as assessed by improvement from baseline in $\mathrm{FEV}_{1}$ versus placebo [36].

Consistent with previous results [37], the data presented here suggest that FF has a wide therapeutic index; the therapeutic dose range was efficacious across individual studies, with a tolerability profile as expected for the class and no evidence of cortisol suppression at the doses assessed.

The strengths of the analyses presented here include the large number of safety end-points assessed, the dataset size (14 studies, 6241 patients) and the length of the studies (up to 76 weeks). A limitation was the disparity in exposure across the treatment groups due to differences in population size and treatment duration, which was mitigated somewhat by the use of exposure-adjusted data.

\section{Conclusions}

In conclusion, this integrated safety analysis demonstrates that the safety profile of FF is consistent with known ICS class effects in patients with asthma, such as local steroid effects, and once-daily FF $100 \mu \mathrm{g}$ and FF $200 \mu \mathrm{g}$ doses are well tolerated in adult and adolescent patients with a range of asthma severities. There was no evidence of cortisol suppression at the doses studied. It should be noted that the safety data has been pooled from different studies and that the individual studies were not designed to identify significant differences regarding specific AEs.

\section{Additional files}

Additional file 1: Table S1. Summary of demographic characteristics and treatment exposure by treatment group (DOCX $41 \mathrm{~kb}$ )

Additional file 2: Figure S1. Plot of pneumonia incidence per 1000 patient-years and $95 \% \mathrm{Cl}$ by treatment group (integrated clinical studies). $B D$, twice daily; $C l$, confidence interval; $F F$, fluticasone furoate; $F P$, fluticasone propionate; OD, once daily. (TIF $465 \mathrm{~kb}$ )

Additional file 3: Figure S2. Plot of serious pneumonia incidence per 1000 patient-years and $95 \% \mathrm{Cl}$ by treatment group (integrated clinical studies). $B D$, twice daily; $C l$, confidence interval; $F F$, fluticasone furoate; $F P$, fluticasone propionate; $O D$, once daily. (TIF $427 \mathrm{~kb}$ )

\section{Abbreviations}

AE: Adverse event; AESI: Adverse event of special interest; $\mathrm{Cl}$ : Confidence interval; $\mathrm{FEV}_{1}$ : Forced expiratory volume in one second; FF: Fluticasone furoate; FP: Fluticasone propionate; HPA: Hypothalamic-pituitary-adrenal; HR: Hazard ratio; ICS: Inhaled corticosteroids; MedDRA: Medical dictionary for regulatory activities; PEF: Peak expiratory flow; SAE: Serious adverse event

\section{Acknowledgements}

The authors thank Leslie Andersen, GSK, for her help in the conception and design of these analyses and interpretation of the data. Editorial support in the form of development of the draft outline and manuscript first draft in consultation with the authors, editorial suggestions to draft versions of this paper, assembling tables and figures, collating author comments, copyediting, fact checking, referencing and graphic services was provided by Jennifer Lawton, PhD and Katherine St. John, PhD at Gardiner-Caldwell Communications (Macclesfield, UK) and was funded by GSK.

\section{Funding}

These analyses were funded by GSK (NCT01499446/FFA20001 [http://www.gskclinicalstudyregister.com/study/FFA20001?study_ids=NCT01499446\#ps], NCT00398645/FFA106783 [http://www.gsk-clinicalstudyregister.com/study/ FFA106783?study_ids=NCT00398645\#ps], NCT00766090/112202 [http:// www.gsk-clinicalstudyregister.com/study/1 12202?study_ids=NCT00766090\#ps], NCT00603746/FFA109684 [http://www.gsk-clinicalstudyregister.com/study/ FFA109684?study_ids=NCT00603746\#ps], NCT00603278/FFA109685 [http:// www.gsk-clinicalstudyregister.com/study/FFA109685?study_ids=NCT00603278\#ps], NCT00603382/FFA109687 [http://www.gsk-clinicalstudyregister.com/study/ FFA109687?study_ids=NCT00603382\#ps], NCT01436071/115283 [http://www.gskclinicalstudyregister.com/study/115283?study_ids=NCT01436071\#ps], NCT01436110/115285 [http://www.gsk-clinicalstudyregister.com/study/ 115285?study_ids=NCT01436110\#ps], NCT01159912/112059 [http://www.gskclinicalstudyregister.com/study/112059?study_ids=NCT01159912\#ps], NCT01431950/114496 [http://www.gsk-clinicalstudyregister.com/study/ 114496?study_ids=NCT01431950\#ps], NCT01165138/HZA106827 [http:/www.gskclinicalstudyregister.com/study/106827?study_ids=NCT01165138\#ps], NCT01086384/106837 [http://www.gsk-clinicalstudyregister.com/study/ 106837?study_ids=NCT01086384\#ps], NCT01134042/HZA106829 [http://www.gskclinicalstudyregister.com/study/106829?study_ids=NCT01134042\#ps] and NCT01244984/1139879 [http://www.gsk-clinicalstudyregister.com/study/ 113989?study_ids=NCT01244984\#ps]).

Availability of data and materials

The datasets supporting the conclusions of this manuscript are included within the article and its additional files.

\section{Authors' contributions}

PMO'B, LJ, CG and WWB contributed to the conception and design of these analyses. CG conducted the data analysis. PMO'B, L, CG, NK, MP, LY and WWB were involved in the interpretation of the data. All authors read and approved the final manuscript.

\section{Competing interests}

PMO'B has acted as a paid consultant to GSK and has received funding for research separate from the work described in this manuscript. $L, C G, N K, M P$ and LY are employees of and shareholders in GSK. WWB has no conflicts to declare.

\section{Ethics approval}

As noted in the Methods section above, all studies complied with the principles of Good Clinical Practice [28] and were approved by relevant Ethics Committees/Institutional Review Boards. Written informed consent was obtained. Studies were conducted in accordance with the applicable version of the Declaration of Helsinki [29]. Regulatory approval was obtained from the relevant health authority where required.

\section{Data availability statements}

Access to the datasets supporting the conclusions of this manuscript may be obtained via https://www.clinicalstudydatarequest.com/. 


\section{Author details}

${ }^{1}$ Michael G. DeGroote School of Medicine, McMaster University, Hamilton, ON, Canada. ${ }^{2}$ Respiratory Medicine Development Centre, GSK, London TW8 9GS, UK. ${ }^{3}$ Quantitative Sciences Division, GSK, London TW8 9GS, UK. ${ }^{4}$ Respiratory Franchise Medical, GSK, London TW8 9GS, UK. ${ }^{5}$ Global Clinical Safety and Pharmacovigilance, GSK, TW8 9GS London, UK. ${ }^{6}$ University of Wisconsin School of Medicine and Public Health, Madison, WI, USA. ${ }^{7}$ Department of Medicine, Michael G. DeGroote School of Medicine, McMaster University, 1280 Main Street West, Room 3W10, Hamilton ON L8S 4K1, Canada.

\section{Received: 7 May 2016 Accepted: 10 November 2016} Published online: 24 November 2016

\section{References}

1. Global Strategy for Asthma Management and Prevention. Global Initiative for Asthma (GINA). http://ginasthma.org/. Accessed 30 Sept 2015.

2. Demoly P, Annunziata K, Gubba E, Adamek L. Repeated cross-sectional survey of patient-reported asthma control in Europe in the past 5 years. Eur Respir Rev. 2012;21:66-74.

3. Price D, Robertson A, Bullen K, Rand C, Horne R, Staudinger H. Improved adherence with once-daily versus twice-daily dosing of mometasone furoate administered via a dry powder inhaler: a randomized open-label study. BMC Pulm Med. 2010;10:1-9.

4. Guest JF, Davie AM, Ruiz FJ, Greener MJ. Switching asthma patients to a once-daily inhaled steroid improves compliance and reduces healthcare costs. Prim Care Respir J. 2005;14:88-98.

5. Salter M, Biggadike $K$, Joyce $L$, et al. Pharmacological properties of the enhanced-affinity glucocorticoid fluticasone furoate in vitro and in an in vivo model of respiratory inflammatory disease. Am J Physiol Lung Cell Mol Physiol. 2007;293:L660-7.

6. Valotis A, Högger P. Human receptor kinetics and lung tissue retention of the enhanced-affinity glucocorticoid fluticasone furoate. Respir Res. 2007:8:54-62.

7. van den Berge M, Luijk B, Bareille P, Dallow N, Postma DS, Lammers JW. Prolonged protection of the new inhaled corticosteroid fluticasone furoate against AMP hyperresponsiveness in patients with asthma. Allergy. 2010;65:1531-5

8. Allen A, Bareille PJ, Rousell VM. Fluticasone furoate, a novel inhaled corticosteroid, demonstrates prolonged lung absorption kinetics in man compared with inhaled fluticasone propionate. Clin Pharmacokinet. 2013;52:37-42.

9. GSK. ARNUITY ELLIPTA US prescribing information. http://www.gsksource. com/pharma/content/dam/GlaxoSmithKline/US/en/Prescribing_Information/ Arnuity_Ellipta/pdf/ARNUITY-ELLIPTA-PI-PIL.PDF. Accessed 30 Sept 2015.

10. GSK. RELVAR ELLIPTA EU summary of product characteristics. http://www. ema.europa.eu/docs/en_GB/document_library/EPAR_-_Product Information/human/002673/WC500157633.pdf. Accessed 22 Fē 2016.

11. GSK. RELVAR ELLIPTA EU prescribing information. https://hcp.gsk.co.uk/ products/relvar.html\#. Accessed 22 Apr 2016.

12. GSK. BREO ELLIPTA US prescribing information. www.gsksource.com/ pharma/content/dam/GlaxoSmithKline/US/en/Prescribing_Information/ Breo_Ellipta/pdf/BREO-ELLIPTA-PI-MG.PDF. Accessed 30 Sept 2015.

13. Medley $\mathrm{H}$, Orozco S, Allen A. Efficacy and safety profile of fluticasone furoate administered once daily in the morning or evening: a randomized, double-blind, double-dummy, placebo-controlled trial in adult and adolescent patients with persistent bronchial asthma. Clin Ther. 2012;34:1683-95.

14. Woodcock A, Bateman ED, Busse WW, et al. Efficacy in asthma of once-daily treatment with fluticasone furoate: a randomized, placebo-controlled trial. Respir Res. 2011;12:132-42.

15. Woodcock A, Bleecker ER, Busse WW, et al. Fluticasone furoate: once-daily evening treatment versus twice-daily treatment in moderate asthma. Respir Res. 2011;12:160-7.

16. Busse WW, Bleecker ER, Bateman ED, et al. Fluticasone furoate demonstrates efficacy in patients with asthma symptomatic on medium doses of inhaled corticosteroid therapy: an 8-week, randomized, placebo-controlled trial. Thorax. 2012;67:35-41.

17. Bleecker ER, Bateman ED, Busse WW, et al. Once-daily fluticasone furoate is efficacious in patients with symptomatic asthma on low-dose inhaled corticosteroids. Ann Allergy Asthma Immunol. 2012;109:353-8.

18. Bateman ED, Bleecker ER, Lötvall J, et al. Dose effect of once-daily fluticasone furoate in persistent asthma: a randomized trial. Respir Med. 2012;106:642-50.
19. O'Byrne PM, Woodcock A, Bleecker ER, et al. Efficacy and safety of oncedaily fluticasone furoate $50 \mathrm{mcg}$ in adults with persistent asthma: a 12-week randomized trial. Respir Res. 2014;15:88-97.

20. Busse WW, Bateman ED, O'Byrne PM, et al. Once-daily fluticasone furoate 50 mcg in mild-to-moderate asthma: a 24-week placebo-controlled randomized trial. Allergy. 2014;69:1522-30.

21. Lötvall J, Bleecker ER, Busse WW, et al. Efficacy and safety of fluticasone furoate $100 \mathrm{mcg}$ once-daily in patients with persistent asthma: a 24-week placebo and active-controlled randomized trial. Respir Med. 2014;108:41-9.

22. Woodcock A, Lötvall J, Busse WW, et al. Efficacy and safety of fluticasone furoate $100 \mathrm{mcg}$ and $200 \mathrm{mcg}$ once daily in the treatment of moderatesevere asthma in adults and adolescents: a 24-week randomized study. BMC Pulm Med. 2014;14:113-22.

23. Bleecker ER, Lötvall J, O'Byrne PM, et al. Fluticasone furoate-vilanterol 100$25 \mathrm{mcg}$ compared with fluticasone furoate $100 \mathrm{mcg}$ in asthma: a randomized trial. J Allergy Clin Immunol Pract. 2014;2:553-61.

24. Bateman ED, O'Byrne PM, Busse WW, et al. Once-daily fluticasone furoate (FF)/vilanterol reduces risk of severe exacerbations in asthma versus FF alone. Thorax. 2014;69:312-9.

25. O'Byrne PM, Bleecker ER, Bateman ED, et al. Once-daily fluticasone furoate alone or combined with vilanterol in persistent asthma. Eur Respir J. 2014:43:773-82.

26. Muraki M, Soutome T, Hashimoto K, Tohda Y. Long-term study of fluticasone furoate/vilanterol combination (FF/ NI) and FF alone in Japanese adult patients with bronchial asthma. Allergol Immunol. 2013;20:110-25.

27. National Institute of Health (NIH). National Asthma Education and Prevention Program, Third Expert Panel on the Diagnosis and Management of Asthma. Expert Panel Report 3: Guidelines for the Diagnosis and Management of Asthma. Bethesda, US Department of Health and Human Services, 2007. http:/www.ncbi.nlm.nih.gov/books/NBK7232/. Accessed 22 Apr 2016.

28. International Conference on Harmonisation (ICH). ICH harmonised tripartite guideline: guideline for good clinical practice E6(R1). http://www.ich.org/ fileadmin/Public_Web_Site/ICH_Products/Guidelines/Efficacy/E6/E6_R1_ Guideline.pdf. Accessed 6 Jan 2015.

29. World Medical Association. Declaration of Helsinki: ethical principles for medical research involving human subjects. http://wma.net/en/30publications/ 10policies/b3/index.html. Accessed 6 Jan 2015.

30. Passalacqua G, Albano M, Canonica GW, et al. Inhaled and nasal corticosteroids: safety aspects. Allergy. 2000;55:16-33.

31. O'Byrne PM, Pedersen S, Carlsson LG, et al. Risks of pneumonia in patients with asthma taking inhaled corticosteroids. Am J Respir Crit Care Med. 2011;183:589-95.

32. Talbot TR, Hartert TV, Mitchel $E$, et al. Asthma as a risk factor for invasive pneumococcal disease. N Engl J Med. 2005;352:2082-90.

33. Busse WW, O'Byrne PM, Bleecker ER, et al. Safety and tolerability of the novel inhaled corticosteroid fluticasone furoate in combination with the $\beta_{2}$ agonist vilanterol administered once daily for 52 weeks in patients $\geq 12$ years old with asthma: a randomized trial. Thorax. 2013;68:513-20.

34. Clark DJ, Grove A, Cargill RI, Lipworth BJ. Comparative adrenal suppression with inhaled budesonide and fluticasone propionate in adult asthmatic patients. Thorax. 1996;51:262-6.

35. Allen A, Schenkenberger I, Trivedi R, et al. Inhaled fluticasone furoate/ vilanterol does not affect hypothalamic-pituitary axis function in adolescent and adult asthma: randomized, double-blind, placebo-controlled study. Clin Respir J. 2013;7:397-406

36. Kempsford RD, Bal J, Baines A, Ravindranath R, Thomas P. The efficacy of fluticasone furoate administered in the morning or evening is comparable in subjects with persistent asthma. Am J Respir Crit Care Med. 2015;191:A4266.

37. Daley-Yates PT. Inhaled corticosteroids: potency, dose equivalence and therapeutic index. Br J Clin Pharmacol. 2015;80:372-80. 